\title{
Estudo descritivo da anatomia do plexo lombossacro em Amazona amazonica
}

\section{(Linnaeus, 1766)}

\author{
Descriptive study of the anatomy of the lumbosacral plexus in Amazona amazonica (Linnaeus, 1766) \\ Estudio descriptivo de la anatomía del plexo lombosacral em Amazona amazonica (Linnaeus, 1766)
}

Recebido: 19/08/2021 | Revisado: 26/08/2021 | Aceito: 31/08/2021 | Publicado: 03/09/2021

\author{
Luan Nascimento Batista \\ ORCID: https://orcid.org/0000-0001-9684-5865 \\ Universidade Federal de Campina Grande, Brasil \\ E-mail: luan.nacimento@hotmail.com \\ Moana Barbosa dos Santos Figuerêdo \\ ORCID: https://orcid.org/0000-0001-5102-5089 \\ Universidade Federal de Campina Grande, Brasil \\ E-mail: moana_figueredo@hotmail.com \\ Joyce Galvão de Souza \\ ORCID: https://orcid.org/0000-0001-5492-6317 \\ Universidade Federal de Campina Grande, Brasil \\ E-mail: joycegalvaosouza@gmail.com \\ Brunna Muniz Rodrigues Falcão \\ ORCID: https://orcid.org/0000-0002-4781-8470 \\ Universidade Federal de Campina Grande, Brasil \\ E-mail: brunnamrfalcao@hotmail.com \\ Ediane Freitas Rocha \\ ORCID: https://orcid.org/0000-0003-4671-3906 \\ Universidade Federal de Campina Grande, Brasil \\ E-mail: edianemedvet@gmail.com \\ Temístocles Soares de Oliveira Neto \\ ORCID: https://orcid.org/0000-0002-5344-8293 \\ Universidade Federal de Campina Grande, Brasil \\ E-mail: temivet@hotmail.com \\ Gildenor Xavier Medeiros \\ ORCID: https://orcid.org/0000-0003-0008-3160 \\ Universidade Federal de Campina Grande, Brasil \\ E-mail: gildenorxavier@gmail.com
}

\begin{abstract}
Resumo
O gênero Amazona compreende os papagaios os quais estão entre as aves mais populares como animais de estimação, justamente por seu hábito dócil e capacidade de reproduzir sons. Possuem pés zigodáctilos, bico curvo e desenvolvido, que conferem a capacidade de escalar árvores e habilidade na manipulação dos alimentos. O presente projeto teve como objetivo descrever a anatomia do plexo lombossacral de 10 (dez) exemplares de papagaio-domangue (Amazona amazonica) doados ao Laboratório de Anatomia Veterinária da Universidade Federal de Campina Grande pelo CETAS/IBAMA-PB. Os animais foram fixados e conservados em solução de formaldeído a $10 \%$ e posteriormente dissecados os nervos do plexo lombossacral. O plexo lombar é formado pelas raízes dos nervos lombares 1, 2 e 3; o plexo sacral é formado pelas raízes dos nervos sacrais 1, 2, 3, 4 e 5, e o plexo pudendo possui 4 origens a partir dos segmentos sacrais da medula espinhal. O plexo lombar após a ramificação, forma dois nervos principais, o cutâneo femoral lateral e o cutâneo femoral cranial. Do plexo sacral surge o nervo isquiático, do qual se originam quatro ramos: nervo tibial, nervo fibular comum, nervo cutâneo femoral caudal e nervo caudal da coxa. O nervo isquiático inerva toda a musculatura que atua nas articulações do joelho e dos dedos. De uma forma geral a origem e os ramos do plexo lombossacral são semelhantes nas aves domésticas.
\end{abstract}

Palavras-chave: Nervo; Papagaio; Psittaciformes.

\section{Abstract}

The amazon genus comprises parrots which are among the most popular birds as pets, precisely because of their docile habit and ability to reproduce sounds. They have zygodactyl feet, curved and developed beak, which give the ability to climb trees and ability to handle food. This project aimed to describe the anatomy of the lumbosacral plexus of 10 (ten) specimens of mangrove parrot (Amazona amazonica) donated to the Veterinary Anatomy Laboratory of the Federal University of Campina Grande by CETAS / IBAMA-PB. The animals were fixed and preserved in $10 \%$ formaldehyde solution and the lumbosacral plexus nerves were subsequently dissected. The lumbar plexus is formed by the roots of the lumbar nerves 1, 2 and 3; the sacral plexus is formed by the roots of the sacral nerves $1,2,3,4$ and 
5 and the pudendal plexus has 4 origins from the sacral segments of the spinal cord. The lumbar plexus after branching forms two main nerves, the lateral femoral cutaneous and the cranial femoral cutaneous. From the sacral plexus arises the sciatic nerve, from which four branches originate: tibial nerve, common fibular nerve, caudal femoral cutaneous nerve and thigh caudal nerve. The sciatic nerve innervates all the musculature that act on the knee and finger joints. In general the origin and branches of the lumbosacral plexus are similar in poultry.

Keywords: Nerve; Parrot; Psittaciformes.

\section{Resumen}

El género Amazonas compreende los loros que se encuentran entre las aves más populares como mascotas, precisamente por su hábito dócil y capacidade para reproducir sonidos. Tienen patas de zygodactyl, um pico curvo y desarrollado, que les da la capacidad de manipular alimentos. Este proyecto tuvo como objetivo describir la anatomia del plexo lumbosacro de 10 (diez) ejemplares de loro de manglar (Amazona amazonica) donados al Laboratorio de Anatomía Veterinaria de la Universidad Federal de Campina Grande por CETAS/ IBAMA-PB. Los animales se fijaron y conservaron em uma solución de formaldehído al $10 \%$ y posteriormente se disecaron los nervios del plexo lumbosacro. El plexo lumbar está formado por las raíces de los nervios lumbares 1, 2 y 3; el plexo sacro está formado por las raíces de los nervios sacros 1, 2, 3, 4 y 5, y el plexo pudendo tiene 4 orígenes em los segmentos sacros de la medula espinal. El plexo lumbar, después de la rama, forma dos nervios principales, el femoral cutâneo lateral y el nervio femoral cutâneo craneal. El nervio ciático surge del plexo sacro, del que se originan cuatro ramas: nervio tibial, nervio peroneo común, nervio cutáneo femoral caudal y nervio caudal del muslo. El nervio ciático inerva toda la musculatura que actúa sobre las articulaciones de la rodilla y los dedos. Em general, el origen y las ramas del plexo lumbosacro son similiares en las aves de corral.

Palabras clave: Nervio, Loro, Psittaciformes.

\section{Introdução}

Nas aves, as adaptações anatômicas para o voo são rígidas, de forma que as variações morfológicas entre todas as espécies de aves são menores do que as observadas nas diversas espécies de mamíferos (Dyce, et al., 2010), são também animais bípedes, com centro de gravidade que se encontra cranial à inserção de seus membros pélvicos (Necker, 2006). E em relação ao plexo lombossacral, há um número pequeno ainda de estudos que o descreva nas diferentes espécies aviárias, quando comparado aos mamíferos (Hussam, et al., 2018), apesar da sua importância, já que seu bom funcionamento é essencial para a correta locomoção terrestre das aves (Necker, 2005), e também age como um órgão sensorial que auxilia na manutenção da postura e da locomoção (Necker, 2006).

Nos psitacídeos os pés são zigodáctilos, apresentando o segundo e o terceiro dedo voltados para frente e o primeiro e quarto para trás. Estas características, juntamente com o bico desenvolvido conferem a capacidade de escalar árvores e habilidade na manipulação dos alimentos (Cubas, et al., 2014). Com relação à funcionalidade diversificada, podemos destacar o plexo lombossacral das aves como sendo muito importante para a apreensão e posterior quebra de alimentos, devido a dinâmica diferenciada de movimentação dos seus membros pélvicos.

O gênero Amazona compreende os papagaios os quais estão entre as aves mais populares como animais de estimação, justamente por seu hábito dócil e capacidade de reproduzir sons. Em território brasileiro são encontradas 11 espécies, dentre elas a Amazona Amazonica (Linnaeus, 1766), popularmente conhecida como curica ou papagaio-do-mangue (Harcourt-Brown, 2009; Péron \& Grooset, 2014).

O estudo descritivo anatômico e morfológico detalhado dos nervos, confere uma poderosa ferramenta para o médico veterinário, no que diz respeito a compreender e melhorar o entendimento acerca das funções de cada nervo, e consequentemente os distúrbios que podem vir a ocorrer na área inervada pelos mesmos. O estudo anatômico dos plexos e consequentemente dos nervos na medicina veterinária já é bastante aprofundado com relação a animais domésticos e vem se tornando mais frequente no âmbito de animais silvestres, vista a necessidade do conhecimento a respeito da anatomia e fisiologia, seja para compreender a sua própria biologia, auxiliando na anatomia comparada ou até mesmo para dar suporte aos profissionais, como médicos veterinários em procedimentos clínicos e cirúrgicos. 


\section{Metodologia}

O presente trabalho foi desenvolvido no Laboratório de Anatomia Veterinária, da Unidade Acadêmica de Medicina Veterinária, do Centro de Saúde e Tecnologia Rural, da Universidade Federal de Campina Grande (LAV/UAMV/CSTR/UFCG), campus de Patos.

Foram utilizados 10 (dez) cadáveres de Papagaio-do-mangue (Amazona amazonica), previamente descongelados, fixados em formaldeído à $10 \%$ através de injeções no sistema vascular, intramuscular e nas cavidades, e em seguida colocados em imersão na mesma solução por tempo mínimo de 48 horas. Após este período, os animais foram lavados com água corrente para retirada do excesso do conservante a fim de possibilitar o manuseio.

Posteriormente os animais foram dissecados com auxílio de instrumental cirúrgico. Primeiramente foram retiradas as penas e em seguida a pele foi rebatida na região sinsacral, tomando-se o cuidado para não danificar os nervos do plexo. Uma vez exposto o plexo, foram dissecadas todas as estruturas que o circundam, para uma melhor visualização da origem dos nervos, e posterior registro escrito e fotográfico de todas as etapas da dissecação. A descrição anatômica foi de acordo com a Nomina Anatomica Avium (I.C.V.G.A.N; W.A.V.A, 2012) e a metodologia científica de acordo com Pereira, et al., (2018).

\section{Resultados e Discussão}

Para expor o plexo lombossacral do papagaio-do-mangue (Amazona amazonica), foi dissecada a região sinsacral, desta forma foram identificados os segmentos lombares e sacrais da medula espinhal. Nesta região surgem os nervos sinsacrais, que são anatomicamente divididos em plexo lombar, plexo sacral e plexo pudendo. O plexo lombar é formado pelas raízes dos nervos lombares 1, 2 e 3, as quais se originam dos segmentos lombares da medula espinhal correspondentes, como mostrado na Figura 1. O plexo sacral, é formado pelas raízes dos nervos sacrais 1, 2, 3, 4 e 5 as quais se originam dos segmentos sacrais da medula espinhal correspondentes, como mostrado na Figura 2. Já o plexo pudendo, por sua vez, possui 4 origens a partir dos segmentos sacrais da medula espinhal, que são os nervos sacrais 5, 6, 7 e 8 (Figura 3), sendo que o primeiro nervo sacral do plexo pudendo possui origem em comum com o último nervo do plexo sacral (Figura 2). 
Figura 1: Vista dorsal do plexo sinsacral de Amazona amazonica com identificação das origens do plexo lombar. 1: Nervo lombar 1; 2: Nervo lombar 2; 3: Nervo lombar 3; L: Plexo lombar; S: Plexo sacral; CG: Corpo gelatinoso.

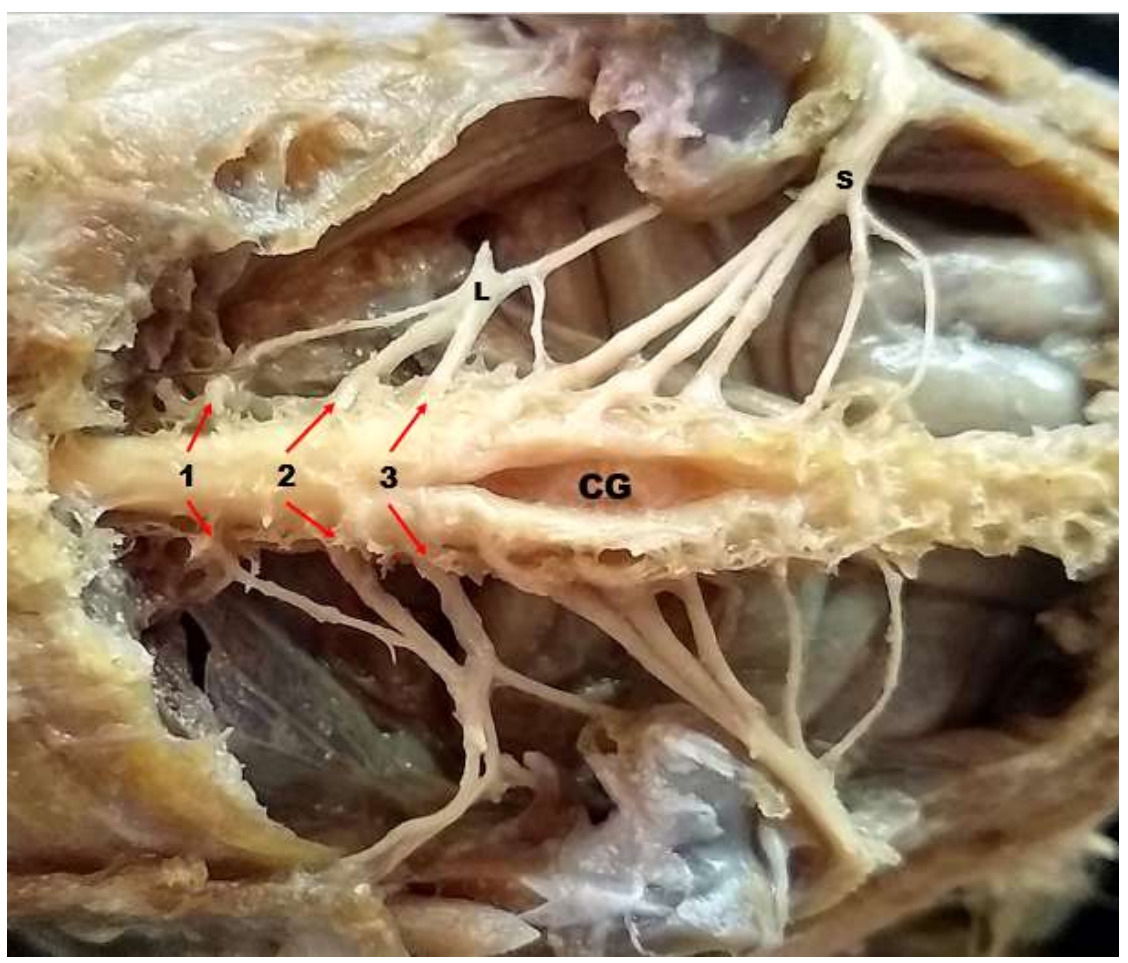

Fonte: LAV/UAMV/CSTR/UFCG.

Figura 2: Vista dorsolateral direita do plexo sacral de Amazona amazonica com identificação das origens do plexo sacral. 1: Nervo sacral 1; 2: Nervo sacral 2; 3: Nervo sacral 3; 4: Nervo sacral 4; 5: Nervo sacral 5; *Origem comum para o último nervo do plexo sacral e primeiro nervo do plexo pudendo (seta branca).

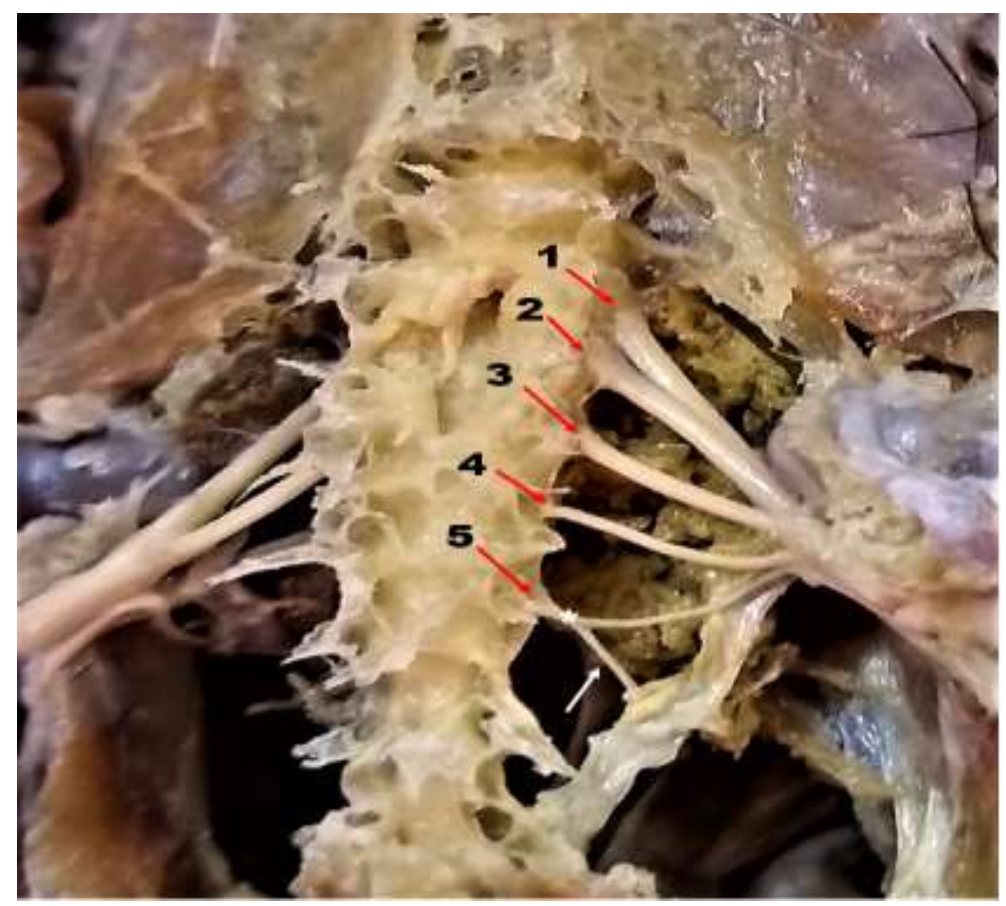

Fonte: LAV/UAMV/CSTR/UFCG. 
Figura 3: Vista dorsolateral direita do plexo pudendo de Amazona amazonica com identificação das origens do plexo pudendo. 5: Nervo sacral 5; 6: Nervo sacral 6; 7: Nervo sacral 7; 8: Nervo sacral 8.

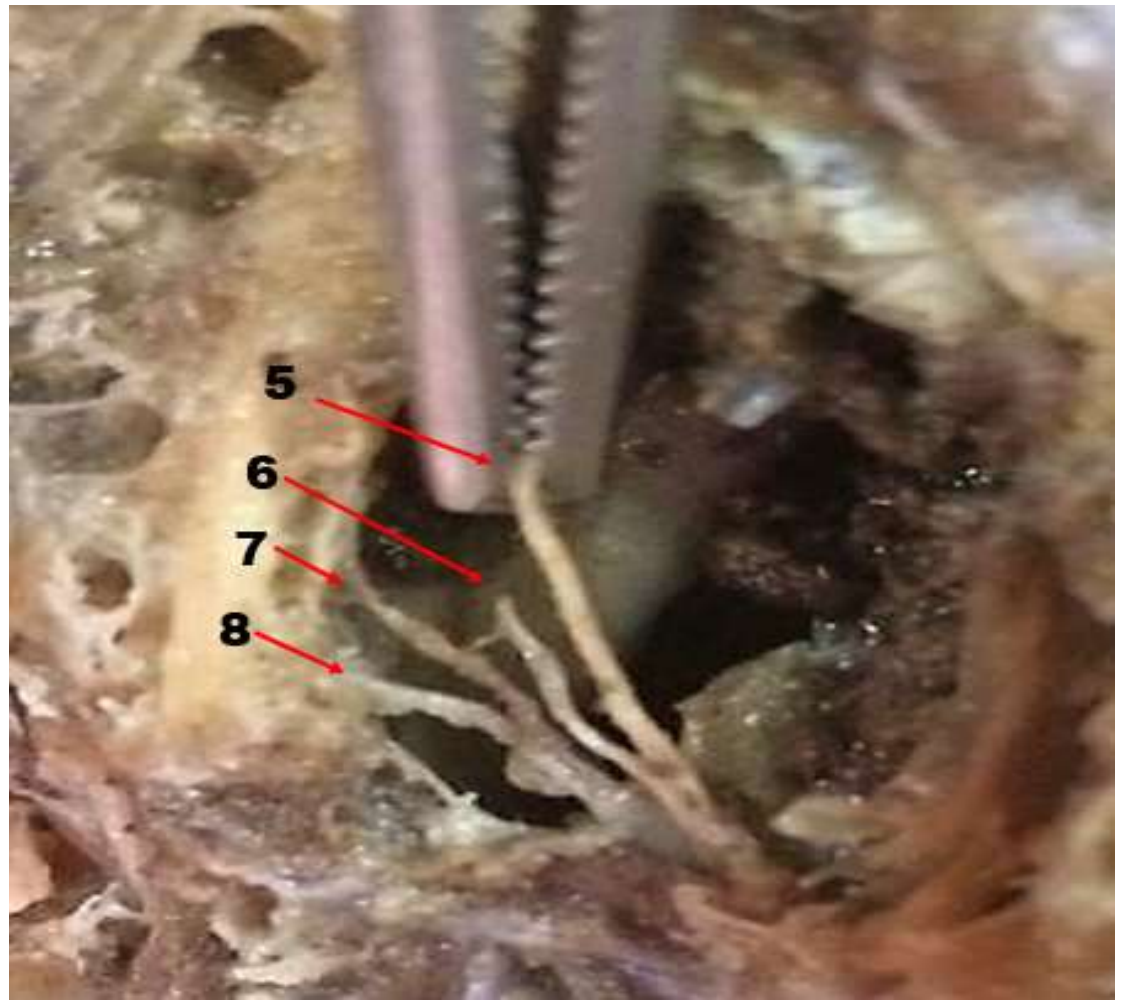

Fonte: LAV/UAMV/CSTR/UFCG.

Balkaya e Ozudogru (2015) observaram que em Accipiter nisus os plexos lombar e sacral tem a mesma origem do Amazoma amazonica, porém Nickel, et al., (1977) e Proctor e Lynch (1993) descreveram que o plexo lombossacral da galinha doméstica (Gallus gallus domesticus) possui uma origem medular a mais. Já no trabalho de Balkaya e Ozudogru (2013), realizado em pombos (Columba livia) foram observadas apenas três origens medulares no plexo sacral, semelhante ao que é descrito também para Coturnix coturnix japonica, também com três ramos (Can, 2011). Mostrando assim que existem diferenças significativas a depender das espécies e funcionalidade do membro pélvico.

O número de origens do plexo sacral do Amazona amazonia também se mostrou semelhante ao observado em Phasianus colchicus mongolicus (Istanbullugil, 2013), enquanto que em Struthio camelus o plexo sacral é constituído dos ramos ventrais do quarto ao décimo nervos espinhais sinsacrais (Honorato, 2017) e em Bubo bubo, do quinto ao nono nervos sacrais (Akbulut, 2016).

O plexo lombar do papagaio-do-mangue (Amazona amazonica), após a ramificação (Figura 4), forma dois nervos principais, o cutâneo femoral lateral e o cutâneo femoral cranial. O nervo cutâneo femoral lateral segue em direção à pele crânio-lateral do fêmur. Já o nervo cutâneo femoral cranial inerva a massa muscular no dorso pré-acetabular do ílio, igualmente observada por Nickel, et al., (1977) e Getty (1986) em galinha doméstica (Gallus gallus domesticus). As origens do plexo lombar também variam entre espécies, sendo no presente estudo observado das raízes dos nervos lombares 1, 2 e 3, enquanto que em Struthio camelus (Mahdy, 2010), foi descrito como sendo sua origem dos nervos 2, 3, 4 e 5, sendo que o quinto nervo também colabora para a formação do plexo sacral e, em Pica pica, o plexo é formado pelos ramos dos nervos dois, três e quatro (Kara \& Özdemir, 2019). 
Do plexo sacral do Amazona amazonica surge o nervo isquiático, formado pela junção de três troncos nervosos. O nervo isquiático entra no forame isquiático localizado na fossa renal caudal, e após este forame o nervo dá origem a quatro ramos: nervo tibial, nervo fibular comum, nervo cutâneo femoral caudal e nervo caudal da coxa (Figura 4).

Foi observado que o nervo tibial é a maior divisão do nervo isquiático, distribuindo-se desde a perna até o pé, e emitindo ramos de inervação para os músculos que atuam nas articulações do joelho. O nervo fibular comum inerva as partes proximais dos músculos craniais da perna.

No trabalho realizado com galinhas domésticas (Gallus gallus domesticus) o nervo fibular comum se dividiu em fibular profundo e fibular superficial no terço médio da perna (Nickel, et al., 1977).

O nervo cutâneo femoral caudal corre em direção caudal e emite ramos nervosos para os músculos e a pele da parte lateral da coxa. Já o nervo caudal da coxa envia ramos para as partes do músculo iliotibial lateral. Já no trabalho de Nickel, et al., (1977), emitem ramos também para os músculos caudiliofemoral e isquiofemoral e se comunica com os ramos cutâneos do plexo pudendo.

Foi observado que as áreas de inervação dos nervos do plexo lombossacral do papagaio-do-mangue (Amazona amazonica) são semelhantes ao descrito por Nickel, et al., (1977) e Proctor e Lynch (1993) na galinha doméstica (Gallus gallus domesticus).

Figura 4: Vista dorsolateral esquerda do trajeto dos nervos do plexo sacral do Amazona amazonica. 1: Ramificações do plexo lombar; 2: Ramos do nervo cutâneo femoral caudal; 3: Nervo caudal da coxa; 4: Músculo iliotibial lateral, parte pré acetabular seccionado para uma melhor visualização das estruturas; 5: Nervo isquiático; 6: Nervo tibial cranial 7: Nervo fibular comum 8: Músculo iliotibial lateral, parte pós acetabular seccionado para uma melhor visualização das estruturas 9: Músculo obturador medial *: Artéria femoral.

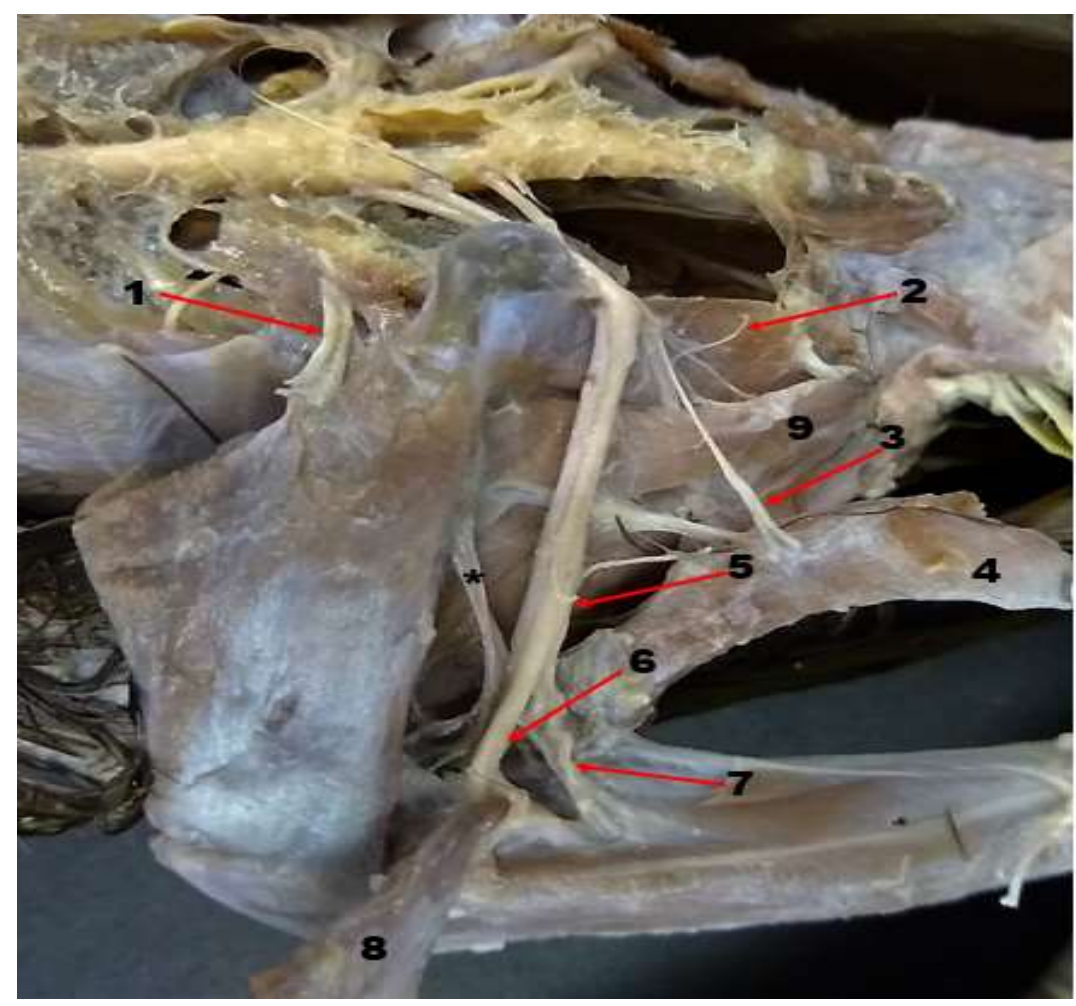

Fonte: LAV/UAMV/CSTR/UFCG. 


\section{Conclusão}

O plexo lombossacral do Amazona amazonica apresenta configuração diferente de outras espécies estudadas, o que pode ser consequência da utilização diferenciada dos membros pélvicos, característica da ordem à qual pertence. Os dados obtidos servem agora de base para médicos veterinários que venham a realizar procedimentos nessa região, tais como anestesias, cirurgias ou até mesmo afim de um diagnóstico clínico mais específico, pois fornece informações anatômicas e morfológicas importantes para tais procedimentos. Esses dados podem também servir para auxiliar em trabalhos futuros que venham a utilizar essas informações a fim de determinar pontos anatômicos para bloqueio anestésico adequado do referido plexo.

\section{Referências}

Akbulut, Y., Demiraslan, Y., Aslan, K. \& Coban, A. (2016). The macroanatomy of the sacral plexus and its nerves in Eurasian Eagle Owls (Bubo bubo). Anatomia, Histologia, Embryologia, 45, 367-372.

Balkaya, H. \& Ozudogru, Z. (2013). Macroanatomic and subgros study on the plexus lumbosacralis and its branches of pigeon (Columba livia). Ataturk Universitesi Veteriner Bilimleri Dergisi, 8.

Balkaya, H., \& Ozudogru, Z. (2015). Macroanatomical aspects of the sacral plexus and its branches in sparrowhawk. Journal of Applied Animal Research, 44 (1), 77-82.

Can, M. \& Özdemir, D. (2011). Macro-anatomic investigations on the plexus lumbosacrales of Japanesse Quail (Coturnix coturnix japonica). Ataturk Universitesi Veteriner Bilimleri Dergisi, 6 (1), 31-45.

Cubas, S. Z., Silva, R. C. J. \& Dias, C. L. J. (2014). Tratado de animais selvagens. Medicina veterinária. (2a ed.), Rocca.

Dyce, K. M., Sack, W. O., \& Wensing, C. J. G. (2010). Tratado de anatomia veterinária. (4a ed.), Elsevier.

Eh-Mahdy, T., El-Nahla, S. M. \& Abbott, L. C., Hassan, S. A. M. (2010). Innervation of the pelvic limbo f the adult ostrich (Struthio camelus). Anatomia, Histologia, Embryologia, 39, 411-425.

Getty, R. (1986). Anatomia dos animais domésticos. (5a ed.), Guanabara Koogan.

Harcour-Brown, N. H. (2002). Orthopedic conditions that affect the avian pelvic limb. Veterinary Clinics of North America: Exotic Animal Practice, 5 (1), 49 81 .

Honorato, A. G. O. (2017). Origem e distribuição dos nervos do plexo lumbosacral da pelve e coxa em avestruz (Struthio camelus).41f. Tese (Doutorado em Ciências veterinárias) - Universidade Federal de Uberândia.

Hussam, M. T., Islam, M. S. \& Alam, J. (2018). Macroanatomical structure of the lumbosacral plexus and its branches in the Indigenous Duck. Bangladesh Veterinary Journal, 52 (1-4): 1-9.

I.C.V.G.A.N - International Committee on Veterinary Gross Anatomical Nomenclature, W.A.V.A. - World Association of Veterinary Anatomists. (2012). Nomina Anatomica Veterinaria. Hannover: Editorial Committee.

Istanbullugil, F. R., Karadag, H., Sefergil, S., Gezer Ínce, N. \& Alpak, H. (2013). Formation of the plexus sacralis in pheasants (Phasianus colchicus mongolicus) and macroanatomic investigation of the nerves originating from the plexus sacralis. Turkish Journal of Veterinary and Animal Sciences, 37 : 160163.

Kara, H. \& Özdemir, D. (2019). Gross anatomy of the lumbar plexus of magpie (Pica pica) and chukar partridge (Alectoris chukar). Turkish Journal of Veterinarya and Animal Sciences, 43: 642-649.

Necker, R. (2006). Specializations in the lumbosacral vertebral canal and spinal cord of birds: evidence of a function as a sense organ which is involved in the control of walking. Journal of Comparative Physiology A: Neuroethology Sensory Neural Behavioral Physiology, 192: 439-448.

Necker, R. (2005). The structure and development of avian lumbosacral specializations of the vertebral canal and the spinal cord with special reference to a possible function as a sense organ of equilibrium. Anatomy and Embryology, 210: 59-74.

Nickel, R., Schummer A. \& Seifirle, E. (1977). Anatomy of the domestic birds. Verlag Paul Parey Berlin.

Pereira, A. S., Shitsuka, D. M., Parreira, F. J. \& Shitsuka, R. Metodologia da Pesquisa Científica. UFSM.

Peron, F. \& Grosset, C. (2014). The diet of adult psittacids: veterinarian and ethological approaches. Journal of Animal Physiology and Animal Nutrition, 98 (3), 1-14.

Proctor, N. S. \& Lynch, P. J. (1993). Manual of ornithology: avian structure and function. Yale University.

Sick, H. (2001). Ornitologia brasileira. Nova Fronteira. 